\author{
A Cascade of Histone Modifications Induces Chromatin \\ Condensation in Mitosis \\ Bryan J. Wilkins et al. \\ Science 343, 77 (2014); \\ DOI: $10.1126 /$ science. 1244508
}

This copy is for your personal, non-commercial use only.

If you wish to distribute this article to others, you can order high-quality copies for your

colleagues, clients, or customers by clicking here.

Permission to republish or repurpose articles or portions of articles can be obtained by following the guidelines here.

The following resources related to this article are available online at www.sciencemag.org (this information is current as of January 23, 2014 ):

Updated information and services, including high-resolution figures, can be found in the online version of this article at:

http://www.sciencemag.org/content/343/6166/77.full.html

Supporting Online Material can be found at:

http://www.sciencemag.org/content/suppl/2013/12/31/343.6166.77.DC1.html

This article cites 33 articles, 14 of which can be accessed free:

http://www.sciencemag.org/content/343/6166/77.full.html\#ref-list-1

This article appears in the following subject collections:

Biochemistry

http://www.sciencemag.org/cgi/collection/biochem 


\section{A Cascade of Histone Modifications Induces Chromatin Condensation in Mitosis}

Bryan J. Wilkins, ${ }^{1}$ Nils A. Rall, ${ }^{1}$ Yogesh Ostwal, ${ }^{2}$ Tom Kruitwagen, ${ }^{3}$ Kyoko Hiragami-Hamada, ${ }^{2}$ Marco Winkler, ${ }^{1}$ Yves Barral, ${ }^{3}$ Wolfgang Fischle, ${ }^{2}$ Heinz Neumann ${ }^{1 *}$

Metaphase chromosomes are visible hallmarks of mitosis, yet our understanding of their structure and of the forces shaping them is rudimentary. Phosphorylation of histone $\mathrm{H} 3$ serine 10 (H3 S10) by Aurora B kinase is a signature event of mitosis, but its function in chromatin condensation is unclear. Using genetically encoded ultraviolet light-inducible cross-linkers, we monitored protein-protein interactions with spatiotemporal resolution in living yeast to identify the molecular details of the pathway downstream of H3 S10 phosphorylation. This modification leads to the recruitment of the histone deacetylase Hst2p that subsequently removes an acetyl group from histone $\mathrm{H} 4$ lysine 16 , freeing the $\mathrm{H} 4$ tail to interact with the surface of neighboring nucleosomes and promoting fiber condensation. This cascade of events provides a condensin-independent driving force of chromatin hypercondensation during mitosis.

$\mathrm{M}$ etaphase chromosome condensation requires the condensin complex, but additional factors must exist because compaction still occurs when condensin is disrupted $(1,2)$. Phosphorylation of histone $\mathrm{H} 3 \mathrm{Ser}^{10}$ (H3 S10) by the kinase Aurora B - an event that correlates with mitosis in all organisms (3) drives hypercondensation of an artificially elongated chromosome during anaphase of budding yeast (4), which suggests that histone tail modifications may influence chromosome condensation. In vitro, the flexible $\mathrm{N}$-terminal domains of the core histones mediate the condensation of arrays of nucleosome core particles upon addition

${ }^{1}$ Free Floater (Junior) Research Group "Applied Synthetic Biology," Institute for Microbiology and Genetics, GeorgAugust University Göttingen, 37077 Göttingen, Germany. 2Laboratory of Chromatin Biochemistry, Max Planck Institute for Biophysical Chemistry, 37077 Göttingen, Germany. Institute of Biochemistry, ETH Zürich, 8093 Zürich, Switzerland.

*Corresponding author. E-mail: hneumann@uni-goettingen.de of $\mathrm{Mg}^{2+}$ ions. Acetylation of histone $\mathrm{H} 4 \mathrm{Lys}^{16}$ (H4 K16) counteracts this process $(5,6)$, but phosphorylation of H3 S10 has no effect (7). The $\mathrm{N}$ terminus of histone $\mathrm{H} 4$ contacts the negatively charged, "acidic" patch formed by the H2A-H2B dimer interface on the surface of a neighboring nucleosome. This interaction is critical for the formation of higher-order chromatin structures $(5,8)$ and has been trapped by the formation of disulfide bridges between suitably installed cysteines $(9,10)$. These observations correlate with defects in the formation of heterochromatin-like structures in yeast caused by mutations in $\mathrm{H} 4 \mathrm{~K} 16$ $(11,12)$. However, all evidence that this interaction mediates chromosome condensation during mitosis is indirect.

Here, we inserted the ultraviolet light (UV)inducible cross-linker amino acid $p$-benzoyl-Lphenylalanine (pBPA) in histone proteins of living yeast, using genetic code expansion $(13,14)$ to trace the dynamic interactions of these proteins along the cell cycle. We expressed Escherichia coli
Tyr-tRNA ${ }_{\text {CUA }}$ and its cognate pBPA-specific tRNA synthetase in Saccharomyces cerevisiae cells (15), leading to the incorporation of pBPA in response to amber codons in an additional, plasmid-borne histone $\mathrm{H} 2 \mathrm{~A}$ gene [tagged with hemagglutinin (HA) and under its native promoter]. Depending on the presence of pBPA in the medium, these cells produce an additional small amount $(\sim 10 \%)$ of the histone containing pBPA at the encoded site (fig. S1). We introduced amber codons at positions 57 to 67,91 , and 92 of S. cerevisiae histone $\mathrm{H} 2 \mathrm{~A}$, which are close to the H4 tail in the crystal structure (16). Cells were irradiated with UV to induce cross-link formation, giving rise to complex but reproducible banding patterns depending on the site of pBPA incorporation (fig. S2). We observed a cross-link product of $\sim 30 \mathrm{kD}$ when $\mathrm{pBPA}$ replaced $\mathrm{Tyr}^{58}$ (Y58), $\mathrm{Ala}^{61}$ (A61), or Glu ${ }^{65}$ (E65) of H2A, which would match the size of HA-tagged H2A cross-linked to H4 (asterisks in fig. S2). Such a cross-link product was readily detected with antibodies to $\mathrm{H} 4$ (anti-H4) in acid extracts of isolated nuclei (Fig. 1A). This band comigrated with the crosslink product detected with antibodies to HA (anti-HA) when analyzed on the same Western blot (Fig. 1B). Deletions in the H4 tail, especially when H4 K16 was affected, strongly diminished cross-linking (Figs. 1C and 2C). Finally, immunoprecipitation of the cross-link products with anti-HA revealed an $\mathrm{H} 4$ cross-reactive band at the expected molecular weight (Fig. 1D). Thus, these experiments identify a cross-link product between $\mathrm{H} 4$ and the acidic patch of H2A in vivo. Surprisingly, the H2A-H4 cross-link is of relatively low abundance compared to other cross-link products. However, cross-linking efficiency is influenced by the chemical environment and may not reflect the relative abundance of different interactions. Accordingly, reconstituted arrays containing pBPA produced the same cross-link-which depended on salt-induced array condensation - with similar efficiency (fig. S3); hence, even under optimal conditions, only a minor fraction formed cross-links.
Fig. 1. Identification of H2A-H4 cross-link products. (A) Western blot analysis (anti-H4) of acid-extracted histones from cells expressing $\mathrm{H} 2 \mathrm{~A}-\mathrm{HA}$ with $\mathrm{pBPA}$ at the indicated positions. (B) H2A-HA Y58pBPA-expressing cells were crosslinked and acid-extracted histones analyzed by SDS-polyacrylamide gel electrophoresis (PAGE). The membrane was cut in half and analyzed separately using either anti-HA or anti-H4. (C) Deletions in the $\mathrm{H} 4$ tail affect the interaction with the acidic patch. Yeast cells carrying genomic mutations in $\mathrm{H} 4$ producing $\mathrm{H} 2 \mathrm{~A}-\mathrm{HA}$ Y58pBPA were arrested with nocodazole. Wholecell extracts were analyzed with anti-HA. (D) Histones extracted from yeasts producing $\mathrm{H} 2 \mathrm{~A}$ HA Y58Y, H2A-HA Y58pBPA, H2A-HA E65Y, or H2A-HA E65pBPA by amber suppression were immunopurified with anti-HA under denaturing conditions and analyzed by Western blot. See figs. S2, S9, and S10 for full-sized Western blots.

\section{A WB:H4}

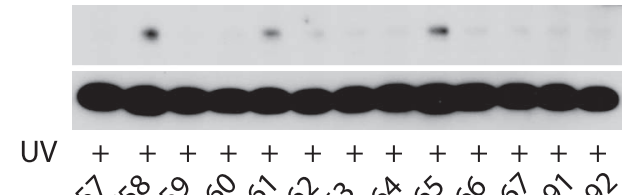

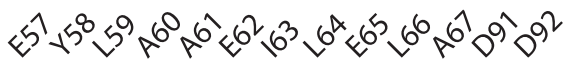

C

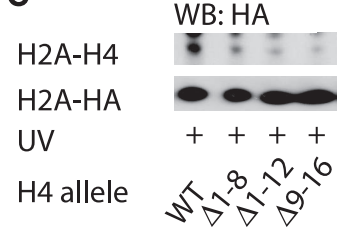

D

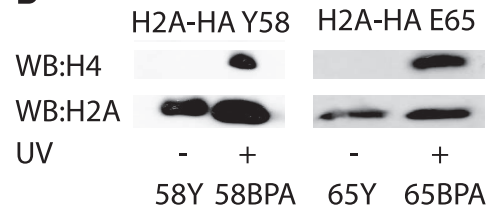

B

$\mathrm{H} 2 \mathrm{~A}-\mathrm{H} 4$

$\mathrm{H} 4$

\section{年}


The H2A-H4 interaction showed a strong dependence on cell cycle stage, with a maximum in $\mathrm{M}$ phase (40 to $80 \mathrm{~min}$ ) coinciding with phosphorylation of H3 S10 (3) (Fig. 2A). H4 K16ac is predominant in S phase (20 min and $100 \mathrm{~min}$ ) and lowest during $\mathrm{M}$ phase, anticorrelating with the intensity of the H2A-H4 cross-link. This indicates that the $\mathrm{H} 2 \mathrm{~A}-\mathrm{H} 4$ interaction, modulated by the acetylation of $\mathrm{H} 4 \mathrm{~K} 16$, may contribute to chromatin condensation in vivo. We observed the same cell cycle-dependent behavior of the crosslink product with anti-H4 in acid extracts of isolated nuclei (Fig. 2B).

The interaction of the $\mathrm{H} 4 \mathrm{~N}$ terminus with the acidic patch is prevented by acetylation of Lys ${ }^{16}$ in vitro $(5,6)$. We therefore tested strains with mutations in the N-terminal tail of $\mathrm{H} 4$ for their ability to produce a H2A-H4 cross-link (Fig. 2C and fig. S4). H4 K16A strongly reduced crosslink formation, in agreement with its effect on the compaction of reconstituted chromatin arrays (5). H4 K16Q had a less pronounced impact, suggesting that glutamine is not a good mimic of acetyl-lysine in this context. In asynchronous H4 $\mathrm{K} 16 \mathrm{R}$ cells, where acetylation is prevented, the interaction was not affected, but in synchronized cells we observed that the H2A-H4 cross-linking efficiency no longer cycled (Fig. 2D). This suggests that $\mathrm{H} 4 \mathrm{~K} 16$ acetylation is a key regulator of chromatin condensation in mitosis.

H3 S10 phosphorylation by Aurora B is a marker of mitosis, but it is unknown whether and how it contributes to chromatin condensation (3). Cells with a H3 S10A mutation showed substantially reduced H2A-H4 cross-linking efficiency, whereas a phospho-mimetic S10D mutation had no effect (Fig. 3A). H4 K16 acetylation anticorrelated with S10 phosphorylation, indicating that the latter acts upstream of H4 K16 deacetylation. In whole-cell lysates of synchronized H3 S10A cells, we found that these defects persist throughout the cell cycle (Fig. 3B). We confirmed these observations by quantifying the abundance of the H2A-H4 cross-link with anti-H4 in a synchronization experiment in which we acid-extracted the histones from isolated nuclei (dotted line in Fig. 3B, lower right; Western blots in fig. S5). In H3 S10D mutants, H4 K16 reacetylation was delayed after mitosis (20- to 60-min time points in Fig. 3B); fluorescence-activated cell sorter (FACS) analysis showed that these cells have difficulties exiting mitosis after release from nocodazole (fig. S6). Hence, the dynamics of these posttranslational modifications must be correctly synchronized to ensure timely formation of the H2A-H4 interaction. A T3A mutation or deletion of four or more residues from the $\mathrm{N}$ terminus of $\mathrm{H} 3$ greatly diminished $\mathrm{H} 2 \mathrm{~A}-\mathrm{H} 4$ cross-linking efficiency, whereas a phospho-mimetic T3D mutation had no effect (Fig. 3A and fig. S7), indicating that phosphorylation of this residue is also involved in the pathway. In fission yeast and vertebrates, phosphorylation of H3 T3 by Haspin kinase recruits the chromosomal passenger complex (CPC), including Aurora $\mathrm{B}$, to the $\mathrm{H} 3$ tail to phosphorylate
H3 S10 (17-19). Indeed, deletion of both Haspin kinase homologs $($ alk1/2 $\Delta)$ attenuated H3 S10 phosphorylation and produced the same downstream effects as a H3 S10A mutation (Fig. 3A).

These findings imply that phosphorylated H3 mediates recruitment of a H4 K16 deacetylase. Indeed, strains deleted for the SIR2 homolog HST2 were unable to deacetylate H4 K16 and to form the H2A-H4 interaction, although $\mathrm{H} 3$ S10 phosphorylation was unaffected (Fig. 3A). Neither H4 K16ac nor the H2A-H4 interaction showed any dependence on the cell cycle in the absence of Hst2p (Fig. 3B). Hence, H3 S10 phosphorylation is required, directly or indirectly, to recruit Hst2p to condensing chromatin. Accordingly, pull-down experiments with synthetic H3 peptides phosphorylated at $\mathrm{Ser}^{10}$ efficiently recovered Hst2p but not Sir2p from whole-cell extracts, relative to a nonphosphorylated control (Fig. 3C).

To test whether Hst2p and H4 K16 deacetylation stimulate chromatin condensation in mitosis, we asked whether Hst2p supports the condensation of the long compound chromosome LC(XII:IV) cen $12 \Delta$, which hypercondenses in anaphase in an

A
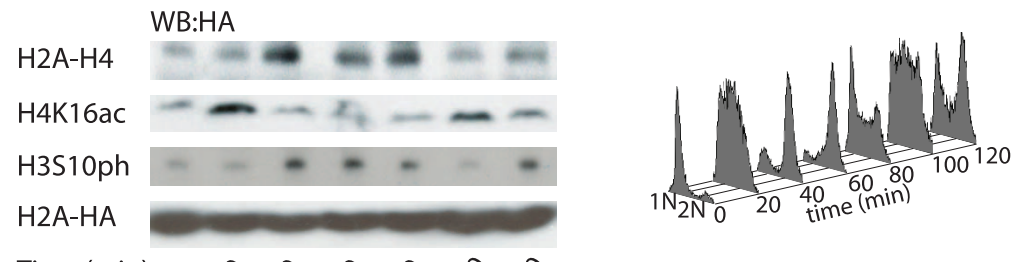

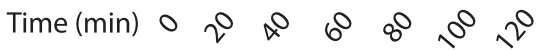

B

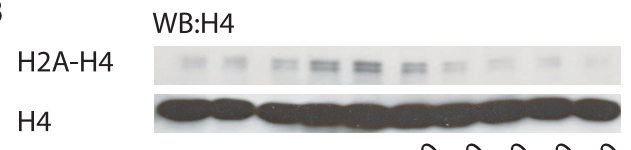

C

Time (min) $02^{\circ} x^{\circ} 6^{\circ} 8^{\circ}, 0^{\circ}, 2^{\circ}, x^{\circ} \leqslant 6^{\circ}, 8^{\circ}$
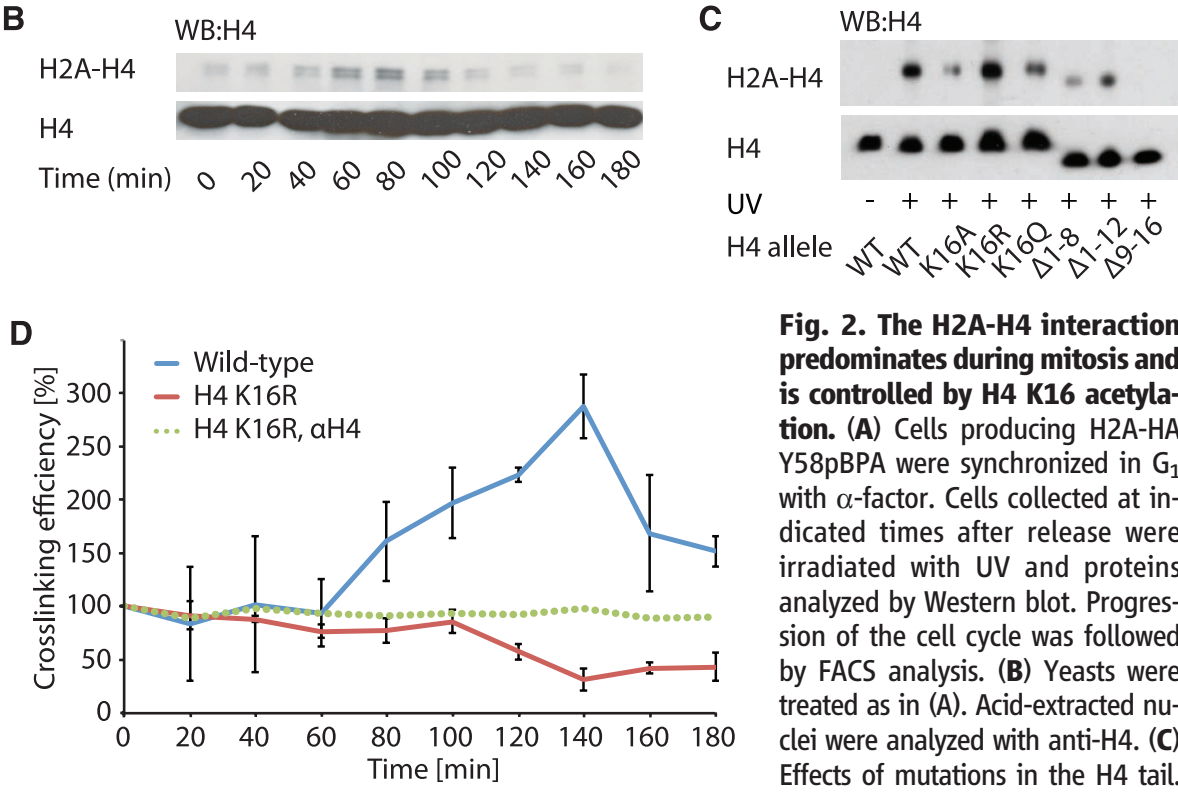

Fig. 2. The H2A-H4 interaction predominates during mitosis and is controlled by H4 K16 acetylation. (A) Cells producing H2A-HA Y58pBPA were synchronized in $G_{1}$ with $\alpha$-factor. Cells collected at indicated times after release were irradiated with UV and proteins analyzed by Western blot. Progression of the cell cycle was followed by FACS analysis. (B) Yeasts were treated as in (A). Acid-extracted nuclei were analyzed with anti-H4. (C) Effects of mutations in the $\mathrm{H} 4$ tail. Yeast cells were treated as in Fig. 1C, and acid-extracted nuclei were analyzed with anti-H4. (D) Nocodazole synchronization of wild-type or H4 K16R mutant yeasts producing H2A-HA Y58pBPA. Cross-linking efficiency was analyzed as in (A) and quantified as percentage of the signal at $t=0$. An identical analysis of acid-extracted nuclei that used anti-H4 to detect the H2A-H4 cross-link is shown by a dotted line. Data are means \pm SD $(n=3)$. See figs. S11 to S14 for full-size Western blots and FACS analysis. 
Unlike in most other organisms (21-23), mutation of H3 S10 does not cause obvious growth defects in yeast (24), but it impairs the ability of fitting the condensation state of chromosomes to spindle length through "adaptive hyperconden- sation" (4). Because budding yeast chromosomes are short, this process is probably not essential for chromosome segregation during most mitoses.

The pathway described here is likely to be more important in higher eukaryotes. Condensa- tion of chromosomes in the absence of condensin has been observed in many different cell types. For example, chicken DT40 cells lacking condensin are able to condense chromatin by an activity termed "regulator of chromosome architecture"
Fig. 3. Phosphorylation of H3 S10 recruits Hst2p to deacetylate $\mathrm{H4} \mathrm{K16}$ and induce the $\mathrm{H} 2 \mathrm{~A}-\mathrm{H} 4$ interaction. (A) Yeasts mutated in $\mathrm{H} 3$ and producing $\mathrm{H} 2 \mathrm{~A}-\mathrm{HA}$ Y $58 \mathrm{pBPA}$ were arrested with nocodazole and irradiated with UV; acid extracts of isolated nuclei were analyzed by Western blot with the indicated antibodies. (B) Mutant yeasts producing H2A-HA Y58pBPA were synchronized with nocodazole and treated as in Fig. 1C. An identical analysis of acid-extracted nuclei that used anti-H4 to detect the H2A-H4 cross-link is shown by a dotted line in the graph at lower right (Western blots in fig. S5). Data are means \pm SD $(n=3)$. (C) Whole-cell extracts from BY4741 cells genomically tagged with green fluorescent protein (GFP) were incubated with immobilized $\mathrm{H} 3$ peptides with or without phosphorylated S10. Eluates were analyzed by Western blot using anti-GFP.

A

A

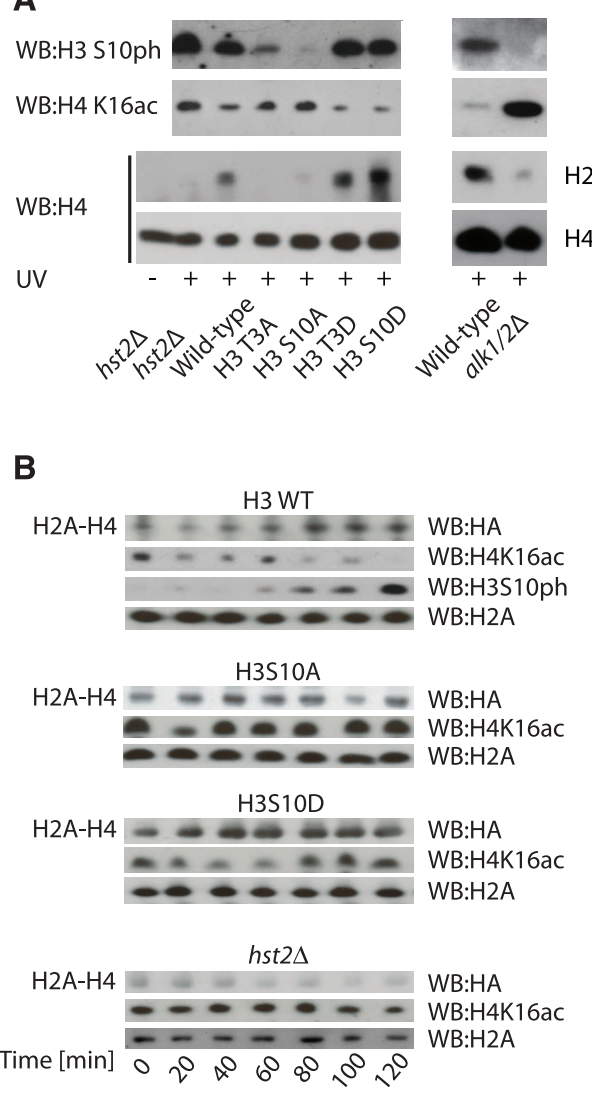

C

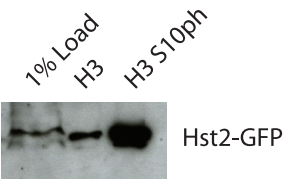

$\mathrm{H} 2 \mathrm{~A}-\mathrm{H} 4$

$\mathrm{H} 4$
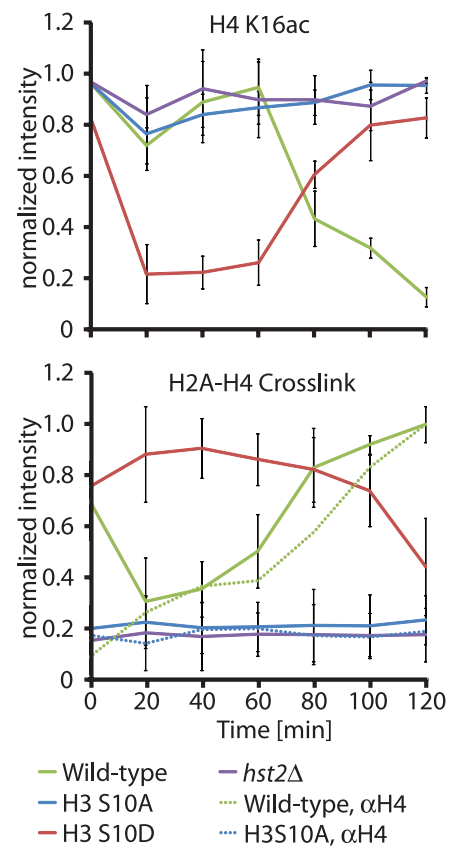

A $\begin{array}{lll}\text { Chr. IV TRP1 } & \text { LYS4 }\end{array}$ LC(XII:IV)cen12 1

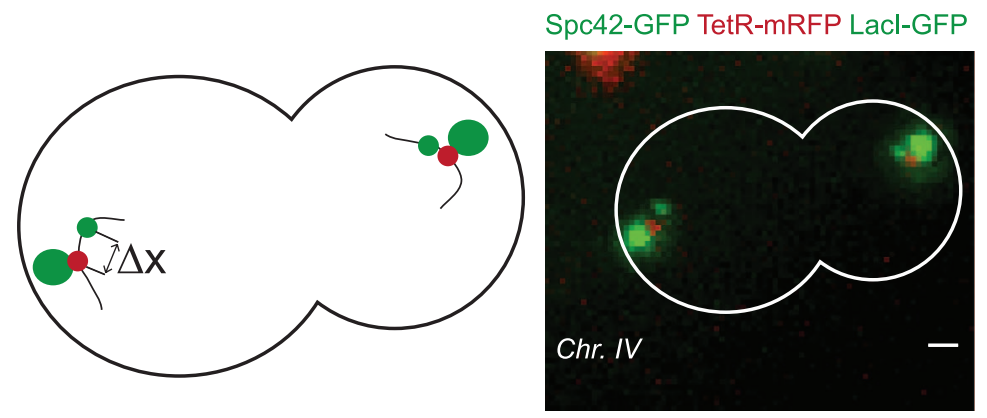

B

Chr. IV $\square L C(X I I: I V) \operatorname{cen} 12 \Delta$

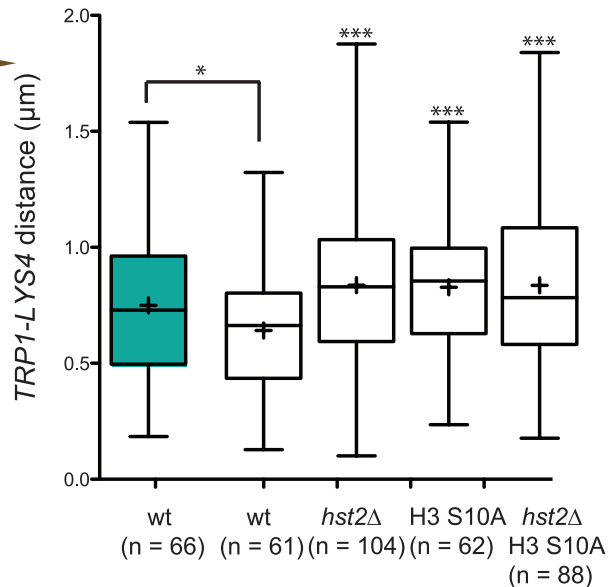

Fig. 4. Phosphorylated H3 S10 and deacetylase Hst2p act in the same pathway to hypercondense long chromosomes. (A) Schematic overview of the wild-type (Chr. IV) and long chromosome (LC(XII:IV)cen12A). A representative anaphase cell contains labeled SPBs (Spc42-GFP; large green dot) and TRP1 and LYS4 loci (smaller red and green dots, respectively). Distance between the latter dots ( $\triangle x$ ) was determined. (B) TRP1-LYS4 distance in yeast mother cells during anaphase for strains of the indicated genotype and containing the indicated chromosome conformation. Boxes include $50 \%$ of data points, whiskers $100 \%$. Medians (lines) and means (plus signs) are shown. ${ }^{*} P<0.05$, ${ }^{* *} P<0.001$. 
(RCA), which is negatively regulated by protein phosphatase 1 (PP1) (25). We postulate that RCA entails the interaction of the $\mathrm{H} 4$ tail with neighboring nucleosomes and that PP1 counteracts this process by dephosphorylating $\mathrm{H} 3 \mathrm{~S} 10$, a known substrate of this enzyme (26). We therefore propose that, together with condensin, the signaling cascade we identified is a conserved mechanism for shaping mitotic chromosomes.

\section{References and Notes}

1. F. Mora-Bermúdez, D. Gerlich, ]. Ellenberg, Nat. Cell Biol. 9, 822-831 (2007).

2. P. Vagnarelli et al., Dev. Cell 21, 328-342 (2011).

3. M. J. Hendzel et al., Chromosoma 106, 348-360 (1997).

4. G. Neurohr et al., Science 332, 465-468 (2011).

5. P. J. Robinson et al., J. Mol. Biol. 381, 816-825 (2008).

6. M. Shogren-Knaak et al., Science 311, 844-847 (2006).

7. C. J. Fry, M. A. Shogren-Knaak, C. L. Peterson, Cold Spring Harb. Symp. Quant. Biol. 69, 219-226 (2004).
8. F. Gordon, K. Luger, J. C. Hansen, J. Biol. Chem. 280, 33701-33706 (2005).

9. B. Dorigo et al., Science 306, 1571-1573 (2004).

10. D. Sinha, M. A. Shogren-Knaak, J. Biol. Chem. 285, 16572-16581 (2010).

11. L. M. Johnson, P. S. Kayne, E. S. Kahn, M. Grunstein Proc. Natl. Acad. Sci. U.S.A. 87, 6286-6290 (1990).

12. E. C. Park, ]. W. Szostak, Mol. Cell. Biol. 10, 4932-4934 (1990).

13. L. Davis, ]. W. Chin, Nat. Rev. Mol. Cell Biol. 13, 168-182 (2012).

14. H. Neumann, FEBS Lett. 586, 2057-2064 (2012)

15. J. W. Chin et al., Science 301, 964-967 (2003).

16. K. Luger, A. W. Mäder, R. K. Richmond, D. F. Sargent, T. ]. Richmond, Nature 389, 251-260 (1997).

17. A. E. Kelly et al., Science 330, 235-239 (2010).

18. F. Wang et al., Science 330, 231-235 (2010).

19. Y. Yamagishi, T. Honda, Y. Tanno, Y. Watanabe, Science 330, 239-243 (2010).

20. C. S. Campbell, A. Desai, Nature 497, 118-121 (2013).

21. K. K. Adhvaryu, E. Berge, H. Tamaru, M. Freitag, E. U. Selker, PLOS Genet. 7, e1002423 (2011).

22. B. G. Mellone et al., Curr. Biol. 13, 1748-1757 (2003).
23. Y. Wei, L. Yu, ]. Bowen, M. A. Gorovsky, C. D. Allis, Cell 97, 99-109 (1999).

24. J. Dai et al., Cell 134, 1066-1078 (2008).

25. P. Vagnarelli et al., Nat. Cell Biol. 8, 1133-1142 (2006)

26. J. Y. Hsu et al., Cell 102, 279-291 (2000)

Acknowledgments: We thank ]. W. Chin for materials. B.J.W. is supported by a postdoctoral fellowship of the Alexander von Humboldt Foundation. Research in the laboratory of H.N. is funded by the German Research Foundation (Emmy-Noether Programme) and the Freefloater Programme of the University of Göttingen. Work in the laboratory of W.F. is supported by the Max Planck Society. K.H.-H. is supported by a Marie Curie Intra-European Fellowship for Career Development (IEF, FP7). T.K. and Y.B. are supported by a grant from the European Research Council to Y.B.

\section{Supplementary Materials}

www.sciencemag.org/content/343/6166/77/suppl/DC1

Materials and Methods

Figs. S1 to S17

Tables $\$ 1$ to $\$ 3$

References (27-34)

9 August 2013; accepted 21 November 2013 $10.1126 /$ science. 1244508

\section{Genetic Screens in Human Cells Using the CRISPR-Cas9 System}

Tim Wang, ${ }^{1,2,3,4}$ Jenny ]. Wei, ${ }^{1,2}$ David M. Sabatini, ${ }^{1,2,3,4,5 *} \dagger$ Eric S. Lander ${ }^{1,3,6 *} \dagger$

The bacterial clustered regularly interspaced short palindromic repeats (CRISPR)-Cas9 system for genome editing has greatly expanded the toolbox for mammalian genetics, enabling the rapid generation of isogenic cell lines and mice with modified alleles. Here, we describe a pooled, loss-of-function genetic screening approach suitable for both positive and negative selection that uses a genome-scale lentiviral single-guide RNA (sgRNA) library. sgRNA expression cassettes were stably integrated into the genome, which enabled a complex mutant pool to be tracked by massively parallel sequencing. We used a library containing 73,000 sgRNAs to generate knockout collections and performed screens in two human cell lines. A screen for resistance to the nucleotide analog 6-thioguanine identified all expected members of the DNA mismatch repair pathway, whereas another for the DNA topoisomerase II (TOP2A) poison etoposide identified TOP2A, as expected, and also cyclin-dependent kinase 6, CDK6. A negative selection screen for essential genes identified numerous gene sets corresponding to fundamental processes. Last, we show that sgRNA efficiency is associated with specific sequence motifs, enabling the prediction of more effective sgRNAs. Collectively, these results establish Cas9/sgRNA screens as a powerful tool for systematic genetic analysis in mammalian cells.

A critical need in biology is the ability to efficiently identify the set of genes underlying a cellular process. In microorganisms, powerful methods allow systematic loss-of-function genetic screening $(1,2)$. In mammalian cells, however, current screening methods fall short-

\footnotetext{
${ }^{1}$ Department of Biology, Massachusetts Institute of Technology (MIT), Cambridge, MA 02139, USA. ${ }^{2}$ Whitehead Institute for Biomedical Research, 9 Cambridge Center, Cambridge, MA 02142, USA. ${ }^{3}$ Broad Institute of MIT and Harvard, 7 Cambridge Center, Cambridge, MA 02142, USA. ${ }^{4}$ David H. Koch Institute for Integrative Cancer Research at MIT, Cambridge, MA 02139 USA. ${ }^{5}$ Howard Hughes Medical Institute, Department of Biology, MIT, Cambridge, MA 02139, USA. 'D Department of Systems Biology, Harvard Medical School, Boston, MA 02115, USA.

*Corresponding author. E-mail: sabatini@wi.mit.edu (D.M.S.); lander@broadinstitute.org (E.S.L.)

†These authors contributed equally to this work.
}

primarily because of the difficulty of inactivating both copies of a gene in a diploid mammalian cell. Insertional mutagenesis screens in cell lines that are near-haploid or carry Blm mutations, which cause frequent somatic crossingover, have proven powerful but are not applicable to most cell lines and suffer from integration biases of the insertion vectors $(3,4)$. The primary solution has been to target mRNAs with RNA interference (RNAi) (5-9). However, this approach is also imperfect because it only partially suppresses target gene levels and can have offtarget effects on other mRNAs, resulting in false negative and false positive results $(10-12)$. Thus, there remains an unmet need for an efficient, large-scale, loss-of-function screening method in mammalian cells.
Recently, the clustered regularly interspaced short palindromic repeats (CRISPR) pathway, which functions as an adaptive immune system in bacteria (13), has been co-opted to engineer mammalian genomes in an efficient manner (14-16). In this two-component system, a single-guide RNA (sgRNA) directs the Cas9 nuclease to cause double-stranded cleavage of matching target DNA sequences (17). In contrast to previous genomeediting techniques, such as zinc-finger nucleases and transcription activator-like effector nucleases (TALENs), the target specificity of CRISPR-Cas9 is dictated by a 20 -base pair (bp) sequence at the $5^{\prime}$ end of the sgRNA, allowing for much greater ease of construction of knockout reagents. Mutant cells lines and mice bearing multiple modified alleles can be generated with this technology $(18,19)$.

We set out to explore the feasibility of using the CRISPR-Cas9 system to perform large-scale, loss-of-function screens in mammalian cells. The idea was to use a pool of sgRNA-expressing lentivirus to generate a library of knockout cells that could be screened under both positive and negative selection. Each sgRNA would serve as a distinct DNA barcode that can be used to count the number of cells carrying it by using high-throughput sequencing (Fig. 1A). Pooled screening requires that single-copy sgRNA integrants are sufficient to induce efficient cleavage of both copies of a targeted locus. This contrasts with the high expression of sgRNAs achieved through transfection that is typically used to engineer a specific genomic change by using the CRISPR-Cas9 system.

We first tested the concept in the near-haploid, human KBM7 CML cell line by creating a clonal derivative expressing the Cas9 nuclease (with a FLAG-tag at its $\mathrm{N}$ terminus) under a doxycyclineinducible promoter (Fig. 1B). Transduction of these cells at low multiplicity of infection (MOI) with a lentivirus expressing a sgRNA targeting the endogenous AAVS1 locus revealed substan- 